Proceedings of SALT 24: 42-62, 2014

\title{
Japanese alternative questions are disjunctions of polar questions*
}

\author{
Wataru Uegaki \\ Massachusetts Institute of Technology
}

\begin{abstract}
We discuss the syntax and semantics of Japanese alternative questions (AltQs), and argue that they are underlyingly disjoined polar questions (PolQs). The argument is based on the following three observations: (i) the minimum syntactic size of a disjunct in a Japanese AltQ is VP or clausal, (ii) sentential operators above the disjunction in an AltQ are never interpreted in both disjuncts, and (iii) the clausefinal particle in an AltQ has to be matched with the particle at the end of the first disjunct. It will be shown that these facts receive straightforward explanations under the analysis that treats Japanese AltQs as disjoined PolQs while they are problematic in a scoping analysis. We also propose a compositional semantics associated with the analysis, and address a potential problem regarding the intervention effect.
\end{abstract}

Keywords: questions, alternative questions, polar questions, disjunction, Japanese

\section{Introduction}

Alternative QUESTIONS (AltQs) are interrogative sentences that involve a disjunction and whose possible answers correspond to the propositional disjuncts of the disjunction, as exemplified in the question-answer pair below. ${ }^{1}$

(1) Q: Do you drink coffee or tea?

A: (I drink) coffee/tea.

Following the Hamblin-style treatment, I assume in this paper that the semantic value of the AltQ in (1) is the set of its possible answers, i.e., (2). ${ }^{2}$

$$
\llbracket(1) \rrbracket=\{\lambda w . \text { I drink coffee in } w, \lambda w . \text { I drink tea in } w\}
$$

* I would like to thank Maria Biezma, Noah Constant, Mitcho Erlewine, Kai von Fintel, Irene Heim, Sabine Iatridou, Andreea Nicolae, David Pesetsky, Floris Roelofsen, Yasutada Sudo and Anna Szabolcsi for valuable comments and suggestions. All errors are my own.

1 The sentence in (1) also has a polar question reading, and the two readings are disambiguated by intonation. Since Japanese, which will be the primary focus of the current paper, does not show a similar effect of intonation, I will ignore the role of intonation throughout this paper.

2 The existence and the uniqueness presuppositions of AltQs will be discussed in section 4.2. 
Japanese alternative questions are disjunctions of polar questions

The task of the compositional semantics of AltQs then is to associate the structure of (1Q) to the answer set in (2) in a compositional fashion.

Although AltQs have been a topic in the semantics of questions since Karttunen 1977, there is relatively little consensus on the proper compositional semantics of AltQs even in the current literature. The present paper concerns one of the current debates in this domain: whether AltQs involve a scope-shifting (hereafter, 'scoping' for short) of the disjunction (Larson 1985; Han \& Romero 2004; Beck \& Kim 2006) or are derived from a disjunction of full polar questions (PolQs) (Pruitt \& Roelofsen 2011; Gračanin-Yuksek 2014), focusing here on AltQs in Japanese in particular.

The claims to be made in this paper is that Japanese AltQs are best analyzed as disjunctions of two PolQs, and that they do not involve any scoping of the disjunction. The argument will be based on the following three observations: (i) the minimum syntactic size of a disjunct in a Japanese AltQ is VP or clausal, (ii) sentential operators above the disjunction in an AltQ are never interpreted in both disjuncts, and (iii) the clause-final particle in an AltQ has to be matched with the particle at the end of the first disjunct.

Three existing analyses of AltQs are presented in the next section, after which Japanese AltQs are introduced in section 3. In this section, I present two problems against the scoping analysis of Japanese AltQs, one concerning the gapping assumed in the analysis along the lines of Han \& Romero 2004 and the other concerning the interaction between the disjunction and other operators. In section 4, I propose an analysis of Japanese AltQs as disjoined PolQs, and discuss the solutions to the problems mentioned in the previous section. Section 5 then discusses a potential problem concerning the intervention effect. Finally, section 6 concludes and discusses the prospects for cross-linguistic typology of AltQs.

\section{Three existing analyses}

In this section, I review three kinds of analyses of AltQs: the scoping analysis, the PolQ disjunction analysis and the scoping-plus-deletion analysis.

\subsection{Scoping analysis}

The basic idea of the scoping analysis is to let the disjunction take scope over the question-forming operator. This scope configuration leads to a question meaning that can be answered by either one of the alternative propositions induced by the disjunction. One of the simplest implementations of this idea is the one using the Karttunen-style semantics. In Karttunen 1977, wh-phrases have the same denotations as existential quantifiers. The difference in meaning between an existential quantifier like someone and a wh-phrase like who is captured by their scopal properties. A wh- 
phrase scopes above the so-called proto-question operator to derive the wh-question interpretation while an existential quantifier always scopes below the proto-question operator.

For an illustration, I give one of the concrete examples of this analysis using Nicolae's (2013) implementation, which follows Heim's (2012) LF-based reformulation of the Karttunen semantics for questions. In this analysis, the AltQ in (1Q) has the LF in (3), where the disjunction coffee or tea is QRed above the Q-operator. ${ }^{3}$

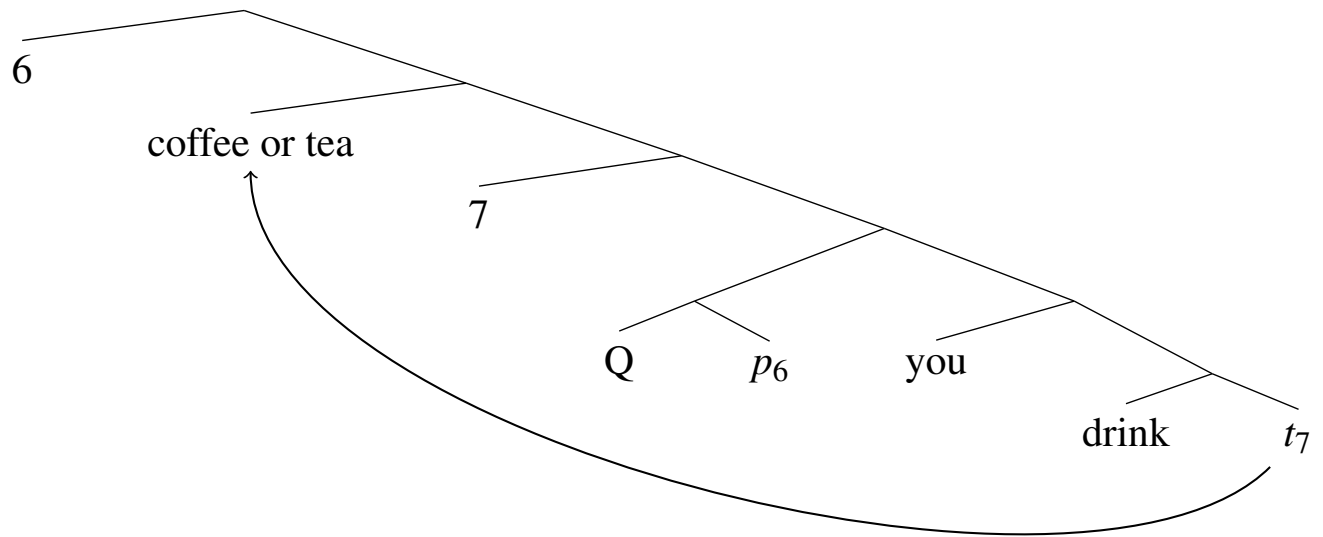

The Q-operator is defined as the identity relation over propositions, as in (4). By giving a generalized quantifier meaning to the disjunction and abstracting over the propositional variable in the sister position of Q, the desired meaning in (6) can be derived. $^{4}$

$$
\begin{aligned}
& \llbracket \mathrm{Q} \rrbracket=\lambda p[\lambda q \cdot p=q] \\
& \llbracket \text { coffee or tea } \rrbracket=\lambda P_{\langle e, t\rangle} \cdot P(\text { coffee }) \vee P(\text { tea }) \\
& \llbracket(3) \rrbracket=\lambda p \cdot[p=\lambda w \cdot \operatorname{like}(\mathbf{j}, \text { coffee }, w) \vee p=\lambda w \cdot \operatorname{like}(\mathbf{j}, \text { tea }, w)]
\end{aligned}
$$

Needless to say, the above formulation is just one of the different formulations of the scoping analysis. In the literature, there are at least two other formulations. One formulation involves an overt movement of whether (Larson 1985; Romero \& Han 2003; Han \& Romero 2004). This analysis follows Larson's idea that whether and either mark the scope position of a disjunction. Romero \& Han (2003) provide a semantic implementation of this analysis by letting whether/either denote the

3 I use the LF notation from Heim \& Kratzer 1998, where binder indices are interpreted as a $\lambda$-abstractor over the co-indexed trace/variable by the rule of Predicate Abstraction.

4 The abstraction of the propositional variable achieves exactly what is obtained by the application of Karttunen's (1977) wh-quantification rule defined as follows.

(i) If $\llbracket \alpha \rrbracket \in D_{\langle e t, t\rangle}$ and $\llbracket \beta \rrbracket \in D_{\langle e,\langle s t, t\rangle\rangle}$, then $\llbracket \alpha \beta \rrbracket=\lambda p . \llbracket \alpha \rrbracket(\lambda x$. $\llbracket \beta \rrbracket(x)(p))$ 
Japanese alternative questions are disjunctions of polar questions

existential closure over choice functions (Reinhart 1997) and the disjunction denote a set of alternative objects which serves as the argument of such a choice function. Under this analysis, when whether is moved above the Karttunen-style Q-operator, the same semantic value as the above analysis is predicted. In the unembedded case, it is assumed that whether is phonetically null.

Another formulation of the scoping analysis is the focus-semantic analysis by Beck \& Kim (2006). This analysis extends Beck's (2006) focus-semantic analysis of $w h$-in-situ to AltQs: Disjunction introduces focus alternatives just like in-situ $w h$ phrases. The focus alternatives are passed up via Point-wise Functional Application (Hamblin 1973) until it meets the Q-operator, which is defined as an operator that 'copies' the focus-semantic value of its prejacent to the ordinary semantic value.

The three different formulations of the scoping analysis make distinct predictions about when an AltQ reading is blocked. For example, the overt movement analysis predicts that an AltQ reading is impossible if the movement of whether violates an island constraint. This is what was claimed to be the case by Larson (1985). On the other hand, the focus semantic analysis predicts that the AltQ interpretation is disrupted when there is an INTERVENER between the Q-operator and the relevant disjunction. Beck \& Kim (2006) claim that the apparent island effect in AltQs are in fact intervention effects, contra Larson (1985). Although the debate over which formulation of the scoping mechanism correctly predicts the detailed generalization is extremely important, I will focus on a different aspect of the analysis of AltQs in this paper: whether a scoping analysis in general is correct in the first place, in light of Japanese AltQs. For this reason, in what follows, arguments for and against the scoping analysis will be formulated in general terms so that they apply to any of the three implementations.

\subsection{PolQ disjunction analysis}

The PolQ disjunction analysis states that AltQs are derived by a disjunction of PolQs, which are themselves syntactically CPs. Thus, the schematic structure of an AltQ in this analysis is the one in (7).

$$
\text { [ [ } \left.\left.\mathrm{CP}_{\mathrm{P}} \mathrm{TP}_{1}\right] \text { Disj [CP Q } \mathrm{TP}_{2}\right] \text { ] }
$$

Under this analysis, what appears to be a coordination of smaller items on the surface involves a deletion in the second CP disjunct. For example, the AltQ in (1) would involve the deletion in (8) under the PolQ disjunction analysis.

(8) Do you drink coffee or do you drink tea?

An important feature of this analysis in the context of the current paper is that it does not involve any scope-shifting operation since the disjunction already scopes 
above the Q-operators, which reside in the complementizer position of each of the PolQs in the structure in (7).

I will discuss the compositional semantics of the PolQ disjunction analysishow the structure in (7) ends up denoting the set of two alternative propositions-in section 4.2 as a part of my discussion of Japanese AltQs. ${ }^{5}$

\subsection{Scoping plus deletion analysis}

The PolQ disjunction analysis has to assume a deletion in the second disjunct in an AltQ with disjuncts smaller than CPs, such as (8). On the other hand, a deletion is not required in a scoping analysis since a scope-shifting mechanism can let the disjunction take scope higher than Q regardless of the disjunction's size (modulo island violations and intervention). However, it is in principle possible to assume both scoping and deletion in AltQs, and this is exactly what Han \& Romero (2004) (hereafter H\&R) propose.

In their analysis, an AltQ interpretation is derived by the overt movement of whether, following Larson 1985 and his arguments based on islands. ${ }^{6}$ Furthermore, they claim that the underlying structure of AltQs involve a disjunction of clausal constituents, and that there is a deletion when the surface string of the disjunction is sub-clausal. H\&R's structure for AltQs is schematically represented in (9) and exemplified with the sentence whether you drink coffee or tea in (10).

$$
\text { [CP whether ...Q...t [TP } \left.\left.1 \text { Disj } \mathrm{TP}_{2}\right]\right]
$$

\section{[CP whether Q $t$ [[you drink coffee] or [you drink tea]]]}

Crucially, although the disjuncts in an AltQ structure in (9) are clausal, they are assumed to be smaller than CPs, as in the above structures.

H\&R's argument for assuming a deletion in AltQs is twofold: one is based on cross-linguistic evidence and the other based on the intonation facts of English AltQs. Here, I will only review the former argument since it is more directly relevant to the current paper. The argument goes as follows: In Hindi, an SOV language, a question involving an object DP disjunction as in (11a) only has a PolQ reading and

5 Indeed, AltQs having the structure of disjoined PolQs exist, as exemplified as follows:

(ii) a. Is it a bird, or is it a plane?

b. John doesn't know [whether it is a bird] or [whether it is a plane].

The question is whether all AltQs have the underlying structure similar to (i).

6 As a compositional-semantic implementation of this, Romero \& Han (2003) use the choice-function analysis sketched in the previous section. 
Japanese alternative questions are disjunctions of polar questions

lacks an AltQ reading. On the other hand, when a disjunct is at least as big as a VP, as in (11b), the question licenses an AltQ reading:

\section{(11) Hindi}

a. Chandra-ne [coffee yaa chai] pii?

Chandra-ERG coffee DISJ tea drink-PFV

'Is it the case that Chandra drank coffee or tea?'

(*AltQ; $\checkmark$ PolQ)

b. [Chandra-ne coffee pii] yaa [Chandra-ne chai pii]?

Chandra-ERG coffee drink.PFV DISJ Chandra-ERG tea drink.PFV

'Did Chandra drink coffee or tea?'

$(\checkmark$ AltQ; $\checkmark$ PolQ)

$H \& R$ argue that the above contrast can best be accounted for when we assume a deletion. Under their analysis, (11a) would require the following deletion in order for it to license an AltQ reading.

(12) [Chandra-ne coffee pii] yaa [Chandra-ne chai pii]?

Chandra-ERG coffee drink.PFV DISJ Chandra-ERG tea drink.PFV

The deletion in the first disjunct in (12) is impossible since there is no backward gapping in Hindi, according to H\&R. Thus, the deletion in (12) is illicit, leading to the prediction that (9) lacks an AltQ reading. On the other hand, in the case of VP/TP disjunction in (11b), an AltQ structure does not involve a backward gapping, meaning that the sentence licenses an AltQ reading. Thus, $H \& R$ conclude that the contrast in (11) is explained if we assume deletion, but is mysterious if an AltQ is derived just by scoping. A scoping mechanism would predict that the disjunction in (11a) can out-scope the Q-operator, just as it did in the analysis of (11b).

\section{Japanese alternative questions}

In the previous section, I reviewed three kinds of compositional semantic analyses of AltQs. As a way to adjudicate between the different analyses from cross-linguistic grounds, I turn to Japanese AltQs in this section.

\subsection{Basic data}

Japanese AltQs are syntactically constrained in the same way as Hindi AltQs: Object DP disjunction does not induce an AltQ reading, as shown in (13a). In contrast, when a disjunct is as big as a VP, the AltQ reading is available, as in (13b). 


\section{Japanese}

a. [Taro-ga [koohii ka ocha]-o non-da-ka] (-ga mondai-da). Taro-NOM coffee DISJ tea-ACC drink-PAST-Q (-NOM question-COP) '(It is a question) whether Taro drank coffee or tea.' (*AltQ; $\checkmark$ PolQ)

b. [Taro-ga [koohii-o non-da-ka ocha-o non-da-ka]]. Taro-NOM coffee-ACC drink-PAST-KA tea-ACC drink-PAST-Q 'whether Taro drank coffee or Tea.' $(\checkmark$ AltQ; ? $\checkmark$ PolQ)

Two notes are in order here. First, the question particle $k a$ is stylistically most natural in an embedded context. For this reason, (13a) is shown with the embedding predicate 'It is a question...'. However, this restriction is only stylistic, and the relevant facts in the embedded clause with $k a$ in principle apply to the matrix case as well. In the examples to follow, the embedding context is omitted for space reasons. Secondly, the disjunction marker $k a$ is homophonous with the question particle $k a$. Thus, in the circumstances where I stay neutral as to whether a certain instance of $k a$ is a question particle or a disjunction marker, I use the neutral gloss KA.

So far, the Japanese data seems to pattern in the same way as the Hindi case, and thus amenable to H\&R's analysis. However, I will provide observations indicating that H\&R's analysis cannot be straightforwardly extended to the Japanese case. The first observation conflicts with H\&R's assumptions about the deletion in AltQs while the latter shows that any kind of scoping operation-whether it is the one in H\&R's analysis or not-is not involved in Japanese AltQs.

\subsection{Problem for H\&R: Backward gapping}

Under H\&R's analysis, to account for the lack of an AltQ reading with an object disjunction, it has to be assumed that the backward gapping in (14) is impossible.

$$
\begin{aligned}
& \text { [[Taro-ga koohii-o non-da] ka, [Taro-ga ocha-o non-da]]-ka. } \\
& \text { Taro-NOM coffee-ACC drink-PAST DISJ Taro-NOM tea-ACC drink-PAST-Q } \\
& \text { 'Did Taro drink coffee or did he drink tea?' }
\end{aligned}
$$

A problem with this assumption is that it is not generally the case that a backward gapping is impossible in Japanese. In the following sentence, where two PolQs are coordinated with the disjunction marker soretomo, a backward gapping is possible.

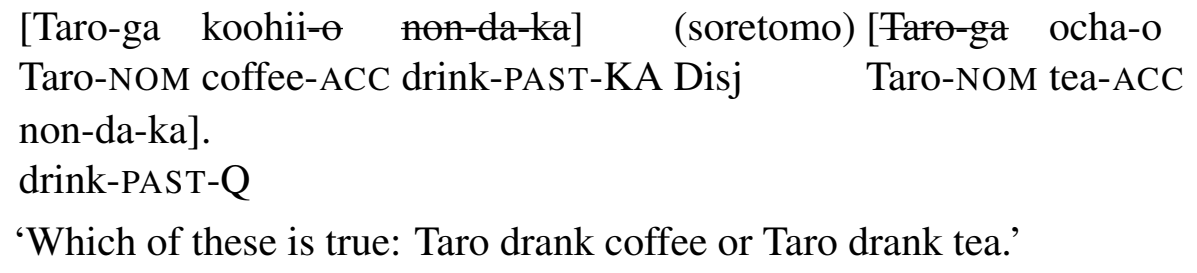


Japanese alternative questions are disjunctions of polar questions

Despite the structural parallel between the gapping in (14) and that in (15), the $\mathrm{H} \& \mathrm{R}$-style analysis has to assume that the former is illicit while the latter is fine. Indeed, (14) and (15) are different in the form of the disjunction markers, $k a$ and soretomo, and this will in fact be crucial for my account in the next section. However, note that the $k a$ in (14) must be a disjunction marker rather than a question particle in the H\&R-style analysis. If it were a question particle, the structure of (14) would not match H\&R's structure for AltQs, where disjuncts are smaller than CPs. Thus, the contrast between (14) and (15) is problematic in the H\&R-style analysis.

\subsection{Problem for scoping: No 'shared' reading of operators}

If an AltQ is derived by scoping, it should be possible for some operator $\alpha$ to be in a position above (the base position of) the disjunction and below the Q-operator in an AltQ. Such a structure will be analyzed as follows in the scoping analyses:

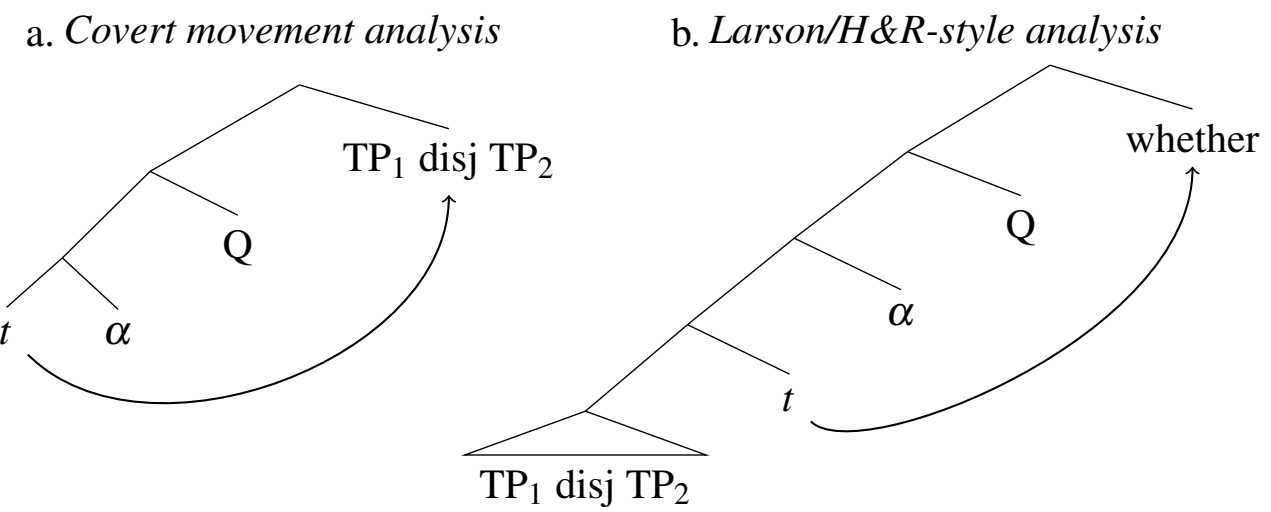

The structures are made head-final to make them compatible with Japanese, and $\alpha$ is the relevant operator sitting between the base position of the disjunction and the Q-operator. Whichever analysis in (16) we choose, the semantic value of the structure will be the interpretation where $\alpha$ is 'shared' in both disjuncts, as shown in the following:

$$
\left\{\llbracket \alpha \rrbracket\left(\llbracket \mathrm{TP}_{1} \rrbracket\right), \llbracket \alpha \rrbracket\left(\llbracket \mathrm{TP}_{2} \rrbracket\right)\right\}
$$

However, it turns out that the reading corresponding to (17) is not available in the sentences that should conform to the structures in (16) in the scoping analysis. In the following sentences, the epistemic modal hazu and the politeness marker desu are immediately followed by the sentence-final question particle $k a$, and correspond to the operator $\alpha$ in (16). Without these operators, the sentence licenses an AltQ reading, as seen in (13b). However, the following sentences lack the relevant AltQ reading where the modal/politeness meaning is 'shared' in both disjuncts. 
(18) Taro-ga koohii-o nomu ka Fare-wa ocha-o nomu-hazu-ka?

Taro-NOM coffee-ACC drink KA tea-ACC drink-must-Q

*'Which is true: Taro must drink coffee or he must drink tea?'

$\checkmark$ 'Is it true that Taro must drink coffee or tea?'

$\checkmark$ 'Which is true: Taro drinks coffee or he must drink tea?'

Taro-wa koohii-o non-da ka Taro-wa ocha-o

Taro-TOP coffee-ACC drink-PAST KA tea-ACC

non-da-no-desu-ka?

drink-PAST-NMNL-POLITE-Q

*'POLITE(Which is true: Taro drank coffee or he drank tea)?'

$\checkmark$ 'POLITE(Is it true that Taro drank coffee or Tea)?'

\#'Which is true: Taro drank coffee or POLITE(he drank tea)?'7

That the relevant operators can appear in a position higher than coordinated TPs can be independently shown in the following TP conjunctions, which indicates that there is no syntactic problem with (18) and (19) having the structure in (16) under the H\&R-style analysis.

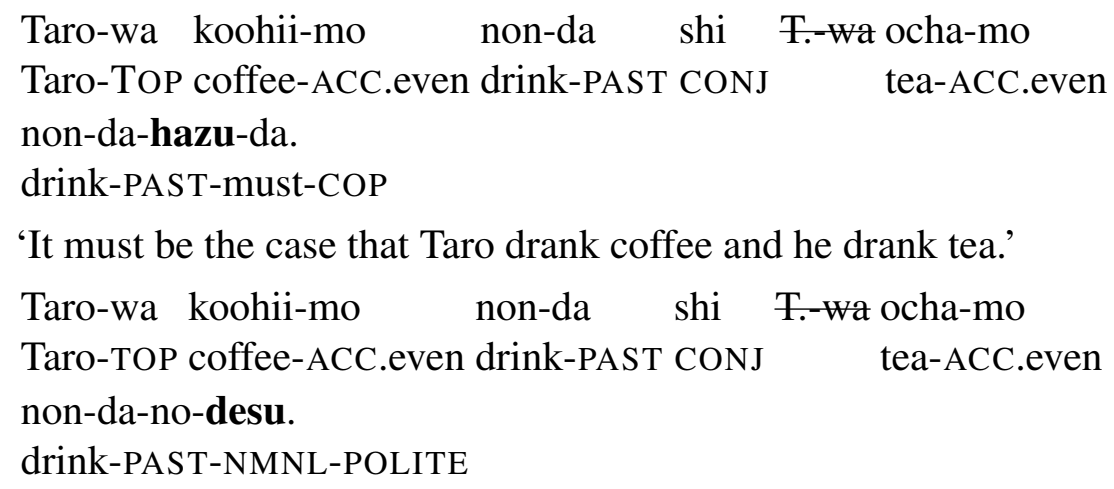

'It must be the case that Taro drank coffee and he drank tea.'

'POLITE(Taro drank coffee and he drank tea)?'

Thus, any kind of scoping analysis-whether it is a H\&R-style analysis or not-predicts that the AltQ readings should be available for (18) and (19) with the interpretation where the modal/politeness operates on both disjuncts. Nevertheless, these readings are not available for (18) and (19). This fact is problematic for any analysis of Japanese AltQs that can make use of a scoping mechanism.

In the rest of this section, I address two possible responses against the above argument. One possible response is to say that the lack of the relevant AltQ readings

7 In contrast to the modal case in (18), the AltQ reading in which the politeness only operates on the second disjunct is infelicitous, presumably for pragmatic reasons: pragmatically, the degree of politeness has to be uniform throughout an utterance. This uniformity is violated in the third reading of (19), which is why the reading is pragmatically infelicitous. 
Japanese alternative questions are disjunctions of polar questions

in (18) and (19) is due to the INTERVENTION EFFECT (e.g., Beck 2006; Beck \& Kim 2006). That is, the modal/politeness operator intervenes in the association between the $\mathrm{Q}$ operator and the disjunction, resulting in the lack of AltQ readings. However, this worry is unwarranted since the operators that cause the effect exemplified in (18) and (19) are not restricted to a certain class identified as interveners in the literature. In fact, the effect is observed in any kind of operator that syntactically resides in the position of $\alpha$ in the structures in (16), including any kind of modal (e.g., beki 'should' (deontic), darou 'be likely' (epistemic)) and evidential markers like reportatives (rashii). Some of these operators are not identified as interveners, nor do they have the general semantic property of interveners proposed in the literature, such as focus-association (Beck 2006) and non-additivity (Mayr 2013).

The second response goes as follows: we could rule out the structure in (16) by positing a syntactic restriction on the minimum size of a disjunct in an AltQ, i.e., that it has to include projections of modals and politeness. Then, (18) and (19) have to be derived from the structure below to receive the intended AltQ interpretation. ${ }^{8}$

$$
[[[\text { TP politeness/modal }] \operatorname{disj}[\text { TP politeness/modal }]] t Q \text { whether }]_{\mathrm{CP}}
$$

Then, it could be further argued that this structure is ruled out due to the constraint against backward gapping (assuming that some solution is given to the problem pointed out in section 3.2). Nevertheless, this restriction on the minimum size of a disjunct is purely stipulative. In fact, under any kind of scoping analysis considered in the previous section (whether it is the covert movement of disjunction, overt movement of whether, or focus-semantic analysis), there is no principled reason to expect that there is a constraint on the minimum size of a disjunct in an AltQ, as suggested above. It would be nicer if we could have a principled explanation for why a disjunct in a Japanese AltQ has to have a certain syntactic size. My account of Japanese alternative questions to be presented below gives an explanation for precisely this question: a disjunct has to be big because it is a disjunction of polar questions, which are syntactically CPs.

\section{Proposal: Japanese AltQs as disjunctions of PolQs}

I propose that the structure of Japanese AltQs is always the one schematized in (23) i.e., disjunction of polar questions.

[ [CP TP 1 Q] Disj [CP $\left.\left.\mathrm{TP}_{2} \mathrm{Q}\right]\right]$

8 The fact that the structure in (22) is possible is not a problem for my argument here, as long as the structure in (16) is possible as well. For, if (16) is possible at all, H\&R should expect the relevant AltQ reading to be possible, contrary to the fact. 
That is, an AltQ reading arises as the result of interpreting (23) as, roughly, 'Is it the case that A, or is it the case that B?'. A more concrete compositional implementation will be given in section 4.2. The disjunction marker in (23) is either covert or realized as soretomo, which we saw in (15). Thus, the structure of a Japanese AltQ under this analysis is exemplified as follows:

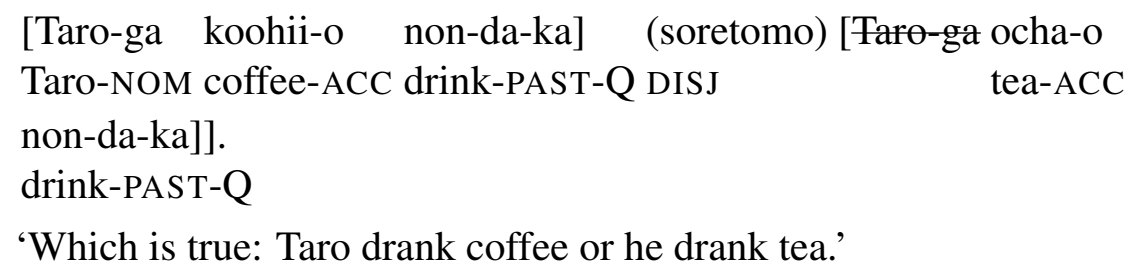

One important claim behind this analysis is that the first $k a$ in (13b) under the AltQ reading is a Q-marker rather than the disjunction marker. This is not to say that all occurrences of the particle $k a$ in Japanese are question particles. As is standardly the case, I assume that there are two kinds of particles pronounced as $k a$ in Japanese: the disjunction marker and the question particle, but my claim here is that the first occurrence of $k a$ in an AltQ should be analyzed as a question particle. This claim is supported by the following fact regarding the correlation between the choice of particles in the end of first disjunct and the possibility of an AltQ reading.

a. Taro-wa koohii-o nonda-no T.-wa ocha-o non-da-no?
Taro-TOP coffee-ACC drink-Q tea-ACC drink-PAST-Q
'Which is true: Taro drank coffee or he drank tea?' (only AltQ)
b. Taro-wa koohii-o nonda-ka T.-wa ocha-o non-da-no?
Taro-TOP coffee-ACC drink-DISJ tea-ACC drink-PAST-Q
'Is it true that Taro drank coffee or tea'

Both questions in (25) use no as their sentence-final particle, ${ }^{9}$ but differ in the particle at the end of the first disjunct. When the first disjunct ends with no, the same particle as the sentence-final one, the sentence only receives an AltQ reading. On the other hand, when the first disjunct ends with $k a$, the sentence only receives a PolQ reading. This fact can only be accounted for if the sentence-internal particle $k a$ in an AltQ is a question particle rather than a disjunction marker, assuming a parallelism requirement between the two question particles in an AltQ, i.e., that the choice of the question particles in the first disjunct and the second has to stay the same. ${ }^{10}$

9 The particle no in questions is syntactically a clausal nominalizer, and no-ending questions are normally analyzed as the shortened form of questions with the no- $k a$ ending. For our purposes, it suffices to assume that no-ending questions are stylistic variant of the $k a$-ending questions without the no- $k a$ sequence (Miyagawa 1987).

10 Although the current analysis proposed here simply stipulates this requirement, it would be nicer if I could derive this requirement from independent grounds. One way to explain it might be to use the 
Japanese alternative questions are disjunctions of polar questions

Since no cannot be a disjunction marker (i.e., there is no disjunction marker in Japanese that is pronounced as no, unlike $k a$ ), (25a) can only be parsed as a sequence of two PolQs. In the current analysis, this structure is correctly interpreted only as an AltQ. On the other hand, in (25b), the sentence-internal $k a$ must be a disjunction marker rather than a question particle due to the above mentioned parallelism requirement. Thus, $(25 \mathrm{~b})$ has a structure in which a single question involves a disjunction, which will only be analyzed as a PolQ in the current analysis. Importantly, this is not the case in a scoping analysis. If scoping the disjunction above the question operator were possible, an AltQ interpretation would be available in (25b) with the sentence-internal $k a$ being analyzed as a disjunction marker.

\subsection{Accounting for the problematic data}

Given the proposal, I can account for the problematic data discussed in sections 3.2 and 3.3, namely the backward gapping and the lack of a shared interpretation of operators.

Backward gapping To remind ourselves of the problem, the H\&R-style analysis has difficulty in accounting for the contrast between (14) and (15).

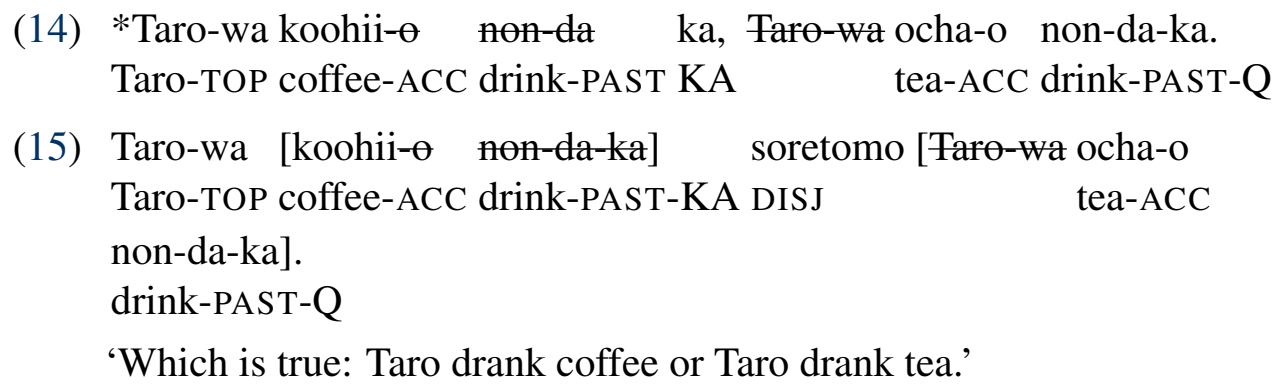

Under the H\&R-style analysis, where the first $k a$ in (14) is a disjunction marker, the gapped materials in (14) and (15) are structurally parallel, so it is unclear why the backward gapping in (15) is allowed while that in (14) isn't.

On the other hand, in the current analysis, the first $k a$ in (14) would have to be a Q-particle rather than a disjunction marker for (14) to be an AltQ. This is so because, if it were a disjunction marker, the first disjunct would lack the question particle, and hence it would not be a PolQ. What this means is that the $k a$ in (14) would have to be a part of the first disjunct, and the gapped material would not be in the right

same kind of pragmatic constraint suggested in footnote 7. Since no and $k a$ differ in their stylistics, AltQs involving two different question particles violate the constraint that requires the degree of casualness to be constant throughout an utterance. 
edge of the first disjunct in an AltQ. This is not the case with the gapping in (15), where the gapping occurs throughout the right edge of the first disjunct.

Now, one can independently show that gapping cannot target a constituent that is not in the right edge of a coordinate in Japanese. Consider the conjunctions below:

a. [Taro-ga doko-e it-ta-ka], sosite [Taro-wa dare-to it-ta-ka]. where-to go-PAST-Q, CONJ who-with go-PAST-Q

b. *[Taro-ga doko-e it-ta-ka], sosite [Taro-wa dare-to it-ta-ka]. where-to go-PAST-Q, CONJ who-with go-PAST-Q

'Where Taro went and with whom he went'

In (26a), the gapping occurs throughout the right edge of the first conjunct while, in (26b), the gapping targets the non-right-edge material it-ta, stranding $k a$. The former gapping is fine, but the latter results in an ungrammatical example. Given this fact, the contrast between (14) and (15) can be accounted for in a similar way: the gapping in (14) is illicit since it targets a non-right-edge material unlike that in (15). Of course, one ultimately needs to understand why this restriction on gapping holds. Although it seems that the restriction makes sense under the Right-Node Raising (RNR) analysis of Japanese gapping (Saito 1987; Koizumi 2000) given the Right Edge Restriction on RNRs (Postal 1974; Sabbagh 2007), I remain neutral as to the proper explanation of the restriction. What is crucial is that the independently observable restriction on Japanese gapping accounts for the contrast in (14) and (15) once one adopts the current analysis.

No shared reading of operators The next problem I address is the problem for scoping analyses in general: sentences such as the following do not have an AltQ reading with the shared reading of an operator (the modal hazu in the case of (18)).

(18) Taro-ga koohii-o nomu ka T.-ga ocha-o nomu-hazu-ka. Taro-NOM coffee-ACC drink KA tea-ACC drink-must-Q

* 'Which is true: Taro must drink coffee or he must drink tea?'

$\checkmark$ 'Is it true that Taro must drink coffee or tea?'

$\checkmark$ 'Which is true: Taro drinks coffee or he must drink tea?'

Under the current analysis, since both disjuncts in an AltQ are underlyingly CPs, they include any projections that are smaller than a $\mathrm{CP}$, including the politeness and the modal projection. Thus, in order for an operator to be interpreted in both disjuncts, it has to be underlyingly present within each disjunct, as below:
[Taro-ga koohii-o nomu hazu-ka] T.-ga ocha-o nomu-hazu-ka]. Taro-NOM coffee-ACC drink must-Q tea-ACC drink-must-Q 
Japanese alternative questions are disjunctions of polar questions

But, for (18) to be derived from this structure, the relevant operator hazu has to be gapped in the first disjunct, as indicated. This violates the right-edge restriction on Japanese gapping. Hence, we correctly predict that the shared reading of operators is impossible in sentences like (18).

\subsection{Compositional semantics}

In this section, I turn to the question of compositional semantics: how does the structure of Japanese AltQs proposed above, as exemplified in (28), receive an appropriate AltQ interpretation?

(28) $[[\mathrm{CP}$ Taro drank coffee-ka] (soretomo) [CP Taro drank tea-ka]].

In order to answer this question, we first need to know what the compositional semantics of PolQs looks like. In this paper, I analyze PolQs as denoting the singleton set of their prejacent proposition, following authors such as Roberts (1996); Gunlogson (2003). Compositionally, this is done simply with the structure in (29) together with the denotation of the Q-operator from Karttunen 1977 in (30) (repeated here from (4)), as shown in (31).

(29) $[\mathrm{CP} \mathrm{Q}[$ Taro drank coffee ] ]

(30) $\llbracket \mathrm{Q} \rrbracket=\lambda p[\lambda q \cdot q=p]$

$$
\llbracket(29) \rrbracket=\lambda q \cdot[q=\lambda w \cdot \operatorname{drank}(\mathbf{t}, \text { coffee }, w)]
$$

Given this semantics for PolQs, (28) gets interpreted with the Generalized Disjunction (Partee \& Rooth 1983) denotation for soretomo in (32). Hence, the desired semantic value of the AltQ (28) is predicted, as in (33).

$$
\begin{aligned}
& \llbracket \text { soretomo } \rrbracket=\lambda Q_{\langle s t, t\rangle} \lambda Q_{\langle s t, t\rangle}^{\prime} \lambda p \cdot Q(p) \vee Q^{\prime}(p) \\
& \llbracket(28) \rrbracket=\{\lambda w \cdot \operatorname{drank}(\mathbf{t}, \operatorname{coffee}, w), \lambda w \cdot \operatorname{drank}(\mathbf{t}, \mathbf{t e a}, w)\}
\end{aligned}
$$

Before concluding the section, I will address why I use the singleton denotation for PolQs rather than somewhat more standard bipolar denotations (Hamblin 1973; Karttunen 1977). In the following, I will discuss how the bipolar answer set can still be derived from the singleton denotation, and defend the singleton analysis. The singleton analysis itself is defended extensively in the literature for reasons related to, e.g., the behavior of answer particles and doubt-type question-embedding predicates (see Biezma \& Rawlins 2012 for a concise review). Here, I will only present an argument for the singleton analysis that is relevant for the proposed analysis of Japanese AltQs. ${ }^{11}$

11 A similar argument for the singleton analysis is made in Biezma \& Rawlins 2012 based on the behavior of English constructions such as Is it a bird, or is it a plane?. 
First, here is how the singleton denotation works in my analysis. I assume that every matrix question comes with an operator which applies to the question denotation and returns a partition over worlds in the sense of Groenendijk \& Stokhof 1984. The operator is defined as in (34) (George 2011). Applying this to the singleton denotation of a PolQ, we derive the usual bipolar answer set, as in (35).

$$
\begin{aligned}
& \llbracket \text { Part } \rrbracket:=\lambda Q_{\langle s t, t\rangle} \cdot\left\{p^{\prime} \mid p^{\prime}=\lambda w \exists w^{\prime}\left[\forall p \in Q\left[p(w)=p\left(w^{\prime}\right)\right]\right]\right\} \\
& \llbracket \text { Part } \rrbracket(\{p\})=\{p, \neg p\}
\end{aligned}
$$

The reason for adopting the singleton analysis has to do with the existence and uniqueness presupposition of AltQs. Just like English AltQs, Japanese AltQs presuppose that one and only one of the alternative propositions is true. This can be shown by the fact that an AltQ of the form in (28) presupposes that Taro drank either coffee or tea and not both, and that a 'both' answer and a 'neither' answer both sound odd unless accompanied with a discourse particle like zitsu-wa 'actually'.

The problem with the bipolar denotation for PolQs is that it cannot account for this presupposition. Suppose the constituent PolQs in (28) had the bipolar denotations. Then, the semantic value of (28) as a result of coordinating the two bipolar denotations by disjunction would consist of four propositions, as follows:

$$
\begin{aligned}
& \{\lambda w \cdot \operatorname{drank}(\mathbf{t}, \operatorname{coffee}, w), \neg \lambda w \cdot \operatorname{drank}(\mathbf{t}, \operatorname{coffee}, w), \lambda w \cdot \operatorname{drank}(\mathbf{t}, \mathbf{t e a}, w), \\
& \neg \lambda w \cdot \operatorname{drank}(\mathbf{t}, \text { tea }, w)\}
\end{aligned}
$$

Given this meaning, there is no way to pick out the two propositions either and only one of which is presupposed to be true by (28), namely $\lambda w$.drank $(\mathbf{t}$, coffee, $w)$ and $\lambda w . \operatorname{drank}(\mathbf{t}, \mathbf{t e a}, w)$.

Importantly, this analysis assumes that Part is not applied to the interrogative CPs that are constituents of an AltQ. That is, I assume the structure as follows.

$$
\text { [Part [[Taro drink coffee Q] Disj [Taro drink tea Q]]] }
$$

Also, the existence and uniqueness presupposition is encoded in the Part operator, using Dayal's (1996) presupposition that the question denotation contains a strongest true member. This is done as follows. ${ }^{12}$

$$
\begin{aligned}
& \left.\llbracket \text { Part } \rrbracket(C)=\lambda Q:|Q|=1 \vee \forall w^{\prime \prime} \in C \exists p \in Q\left[p\left(w^{\prime \prime}\right) \wedge \forall p^{\prime} \in Q\left[p^{\prime}\left(w^{\prime \prime}\right) \rightarrow p \subseteq p^{\prime}\right]\right]\right] . \\
& \left\{p \mid p=\lambda w \exists w^{\prime}\left[\forall p^{\prime} \in Q\left[p^{\prime}(w)=p^{\prime}\left(w^{\prime}\right)\right]\right]\right\}
\end{aligned}
$$

12 In (38), $C$ is the context set under which the question is evaluated. Also, if $Q$ is a singleton, (38) does not require that there be a true strongest answer in the set. This exception makes the treatment of uniqueness presupposition compatible with my analysis of PolQs. If it were not for this exception, Part would be defined for $\{p\}$ only if $p$ is true. 
Japanese alternative questions are disjunctions of polar questions

\section{Intervention effect?}

In this section, I address a potential challenge for the current analysis of Japanese AltQs posed by Beck \& Kim's (2006) report that Korean AltQs are subject to intervention effects:

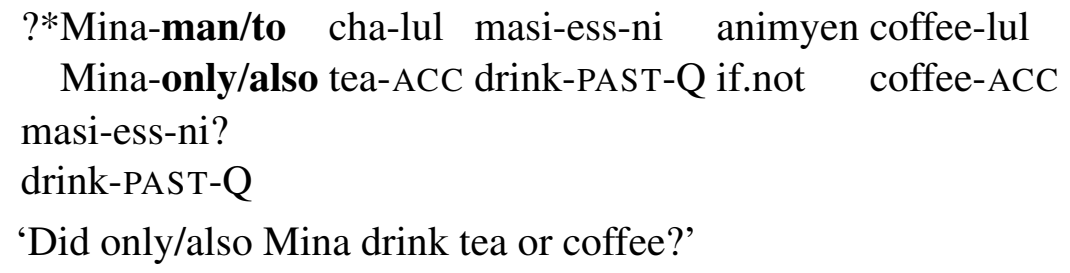

Below are the Japanese counterparts of these examples, with the author's judgments.
a. ? Taro-dake-ga ocha-o nonda-no soretomo koohii-o nonda-no? Taro-only-NOM tea-ACC drank-Q DIS J coffee-ACC drank-Q
'Did only Taro drink tea or coffee'

\section{b. (?) Taro-mo ocha-o nonda-no soretomo koohii-o nonda-no? Taro-also tea-ACC drank-Q DISJ coffee-ACC drank-Q \\ 'Did also Taro drink tea or coffee?'}

The intervention effect in AltQs can be accounted for in the focus-semantic analysis of AltQs (Beck \& Kim 2006), combined with the focus-based explanation of intervention effects (Beck 2006). On the other hand, the same explanation of intervention effects is not available in the current analysis since it should be possible to disjoin CPs each of which involve an intervener. However, an alternative explanation is possible: it can be attributed to a constraint on the deletion operation. Specifically, deriving an AltQ with the surface structure with the intervening focus-sensitive operator would violate the Focus Deletion Constraint (FDC) i.e., a constraint against the ellipsis of a focused constituent. ${ }^{13}$ (Heim 1997; see Pruitt \& Roelofsen 2011 for an account of English intervention effect in AltQs in terms of FDC).

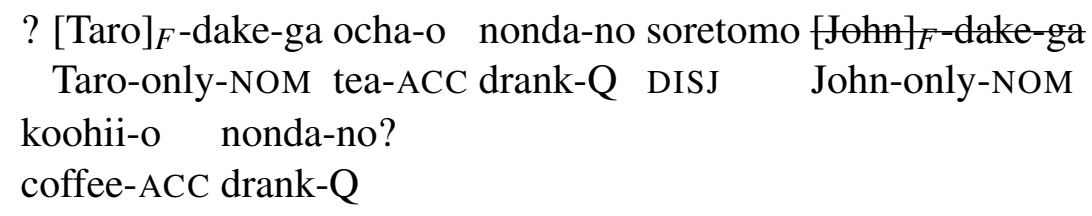

'Did only Taro drink tea or coffee'

13 More precisely, FDC bans the deletion of focused material except when it is deleted with its associated $\sim$ operator and $C$. So, I am assuming here that $\sim$ and $C$ associated with dake 'only' is outside the ellipsis site, as follows.

(iii) ?[[Taro $]_{F}$-dake $\left(C_{1}\right)$-ga ocha-o non-da] $\sim \mathrm{C}_{1}$-no soretomo [[Taro $]_{F}$-dake $\left(C_{2}\right)$-ga koohii-o Taro-only-NOM tea-ACC drink-PAST-Q DISJ Taro-only-NOM coffee-ACC non-da] $\sim \mathrm{C}_{2}$-no? drink-PAST-Q 
In this analysis, we predict that the sentence becomes grammatical if an intervener is not associated with focused material in the elided position. This is exactly what happens in (42) below. In (42), it is shown that the adverb 'often', which acts as an intervener in a language like German (as in (43)), does not make a Korean/Japanese AltQ ungrammatical when it intervenes between the Q operator and the disjunction.

a. Mina-ka cacwu John-ul phathi-ey chotayha-ess-ni animyen Mina-NOM often John-ACC party-to invite-PAST-Q if.not

Mina-ka eacwu Bill-ul phathi-ey chotayha-ess-ni?

(Korean)

Bill-ACC party-to invite-PAST-Q

b. Mina-wa yoku John-o paati-ni shootai-sita-no soretomo Mina-wa Mina-TOP often John-ACC party-to invite-PAST-Q if.not yoku Bill-o paati-ni shootai-sita-no?

(Japanese)

Bill-ACC party-to invite-PAST-Q

'Did Mina often invite John or Bill to the party?'

\# Hat Peter oft Kaffee getrunken oder Kuchen gegessen?

(German) has Peter often coffee drunk or cake eaten

'Did Peter often drink coffee or eat cake?'

The grammaticality of the examples in (42) is expected in the current analysis since the elided material does not contain a focus to be associated with 'often'. ${ }^{14}$

\section{Conclusions and cross-linguistic prospects}

In this paper, I discussed the syntax and semantics of Japanese alternative questions (AltQs), and argued that they are underlyingly disjoined polar questions (PolQs). The facts regarding the backward gapping and the shared interpretation of sentential operators are problematic for the Han \& Romero-style analysis or a scoping analysis in general. On the other hand, the current analysis provides straightforward solutions to these problems. Furthermore, the fact regarding the matching requirement between the clause-final question particle and the particle at the end of the first disjunct receives a natural account under the current proposal, while it remains puzzling in a scoping analysis that analyzes the latter particle as a disjunction marker. Thus, the current analysis contributes to the current debate over the proper syntax/semantics of AltQs by providing a concrete case study showing that AltQs in a particular language are always disjoined PolQs, along with the analysis of Turkish AltQs by Gračanin-Yuksek (2014).

14 It should be noted that this leaves open the question of why the same kind of potential interveners like 'often' do not give rise to the intervention effects in Korean in-situ wh-questions. 
Japanese alternative questions are disjunctions of polar questions

Needless to say, this paper says little about AltQs in languages other than Japanese. The current study has to be ultimately embedded in a larger typological study in order for us to understand the cross-linguistic universal of AltQ syntax/semantics. One possibility of such a typology involves exploring the scopal property of disjunctions. The current analysis of Japanese AltQs indicates that the Japanese disjunction never scopes above the Q-operator. As such, the only way to derive AltQs in Japanese is to disjoin PolQs. By analogy to the scopal property of existential quantifiers like someone in Karttunen 1977, I label this scopal feature as [-wh]. That is, both someone and the Japanese disjunction $k a$ cannot out-scope $\mathrm{Q}$, being [-wh] items. In contrast, a [+wh] disjunction always out-scopes the Qoperator, just like who in Karttunen's question semantics (see Nicolae 2013 for a similar suggestion). In this picture, the situation in English could be analyzed as allowing the disjunction to scope above or below the Q-operator, where the former leads to an AltQ interpretation while the latter to a PolQ interpretation. In other words, the English disjunction is [ \pm wh]. This typology can be extended to languages that possess two disjunction markers, such as Basque and Arabic. In these languages, one disjunction can be analyzed as [+wh], which forces an AltQ reading while the other can be analyzed as [-wh], which never appears in an AltQ. In Uegaki 2014, I discuss the typology of disjunctions along these lines more closely, and show that the empirical picture is not as simple as what I have just described.

Another research question is how the current analysis can be integrated to the recent projects in which the two uses of $k a$, i.e., as the disjunction marker and as the question particle (or similar morphemes in other languages such as Sinhala and Slavic languages) are semantically unified (Slade 2011; Szabolcsi 2014a,b; Mitrovič \& Sauerland 2014). One of the challenges for such an integration is that it is crucial in the current analysis that the $k a$ coordinating DPs in (44) below only functions as a disjunction marker, rather than a generalized question-forming operator, to account for its lack of an AltQ reading in contrast to (13b).

Taro-ga [koohii ka ocha-ka]-o non-da.

Taro-NOM coffee KA tea-KA-ACC drink-PAST

'Taro drank either coffee or tea.'

(*AltQ)

(13b) Taro-ga koohii-o non-da-ka ocha-o non-da-ka.

Taro-NOM coffee-ACC drink-PAST-KA tea-ACC drink-PAST-KA

'whether Taro drank coffee or Tea'

$(\checkmark$ AltQ; ? $\checkmark$ PolQ)

If we are to unify the two uses of $k a$, it remains to be answered why the size of disjuncts matters in whether $k a$ can induce an AltQ reading, as shown in the contrast above. The semantic unification of the $k a$-type morphemes faces other non-trivial problems as well, and I have to leave this issue for future studies. 


\section{References}

Beck, Sigrid. 2006. Intervention effects follow from focus interpretation. Natural Language Semantics 14(1). 1-56.

Beck, Sigrid \& Shin-Sook Kim. 2006. Intervention effects in alternative questions. Journal of Comparative Germanic Linguistics 9. 165-208.

Biezma, Maria \& Kyle Rawlins. 2012. Responding to alternative and polar questions. Linguistics and Philosophy 35(5). 361-406.

Dayal, Veneeta. 1996. Locality in WH Quantification: Questions and Relative Clauses in Hindi. Dordrecht: Kluwer Academic Publishers.

George, Benjamin. 2011. Question embedding and the semantics of answers: UCLA $\mathrm{Ph}$. D. dissertation.

Gračanin-Yuksek, Martina. 2014. Alternative questions in Turkish. Paper presented at Workshop in Altaic Formal Linguistics (WAFL) 10, May 2014, MIT.

Groenendijk, Jeroen \& Martin Stokhof. 1984. Studies on the semantics of questions and the pragmatics of answers: University of Amsterdam Ph. D. dissertation.

Gunlogson, Christine. 2003. True to Form: Rising and Falling Declaratives as Questions in English. New York: Routledge.

Hamblin, Charles L. 1973. Questions in Montague English. Foundations of Language 10(1). 41-53.

Han, Chung-Hye \& Maribel Romero. 2004. The syntax of whether/Q.. or questions: Ellipsis combined with movement. Natural Language and Linguistic Theory 22(3). 527-564.

Heim, Irene. 1997. Predicates or formulas? Evidence from ellipsis. In Aaron Lawson (ed.), Semantics and Linguistic Theory (SALT) 7, 197-221. Ithaca, New York: CLC Publications.

Heim, Irene. 2012. Lecture notes on questions. Ms., Massachusetts Institute of Technology.

Heim, Irene \& Angelika Kratzer. 1998. Semantics in Generative Grammar. Oxford: Blackwell.

Karttunen, Lauri. 1977. Syntax and semantics of questions. Linguistics and Philosophy 1(1). 3-44.

Koizumi, Masatoshi. 2000. String vacuous overt verb raising. Journal of East Asian Linguistics 9. 227-285.

Larson, Richard K. 1985. On the syntax of disjunction scope. Natural Language and Linguistic Theory 3(2). 217-164.

Mayr, Clemens. 2013. Intervention effects and additivity. Journal of Semantics Advance Access.

Mitrovič, Moreno \& Uli Sauerland. 2014. Decomposing coordination. Paper presented at North East Linguistic Society (NELS) 44, Oct. 18-20, 2013, University 
Japanese alternative questions are disjunctions of polar questions

of Connecticut.

Miyagawa, Shigeru. 1987. LF affix raising in Japanese. Linguistic Inquiry 18(2). 362-367.

Nicolae, Andreea Cristina. 2013. Any questions?: Polarity as a window into the structure of questions: Harvard University Ph. D. dissertation.

Partee, Barbara H. \& Mats Rooth. 1983. Generalized conjunction and type ambiguity. In Rainer Bäuerle, Christoph Schwarze \& Arnim von Stechow (eds.), Meaning, Use, and Interpretation of Language, 361-383. Berlin: Walter de Gruyter.

Postal, Paul M. 1974. On Raising. Cambridge, MA: The MIT Press.

Pruitt, Kathryn \& Floris Roelofsen. 2011. Disjunctive questions: Prosody, syntax, and semantics. Ms., University of Massachusetts, Amherst and ILLC, University of Amsterdam.

Reinhart, Tanya. 1997. Quantifier scope: How labor is divided between QR and choice functions. Linguistics and Philosophy 20(4). 335-397.

Roberts, Craige. 1996. Information structure in discourse: Towards an integrated formal theory of pragmatics. In Jae-Hak Yoon \& Andreas Kathol (eds.), $O S U$ Working Papers in Linguistics, No. 49: Papers in Semantics, The Ohio State University.

Romero, Maribel \& Chung-Hye Han. 2003. Focus, ellipsis and the semantics of alternative questions. In Claire Beyssade, Olivier Bonami, Patricia Cabredo Hofherr \& Francis Corblin (eds.), Empirical Issues in Formal Syntax and Semantics 4, 291-307. Presses Universitaires de Paris-Sorbonne.

Sabbagh, Joseph. 2007. Ordering and linearizing rightward movement. Natural Language and Linguistic Theory 25(2). 349-401.

Saito, Mamoru. 1987. Three notes on syntactic movement in Japanese. In Takashi Imai \& Mamoru Saito (eds.), Issues in Japanese Linguistics, Dordrecht Reidel.

Slade, Benjamin. 2011. Formal and philological inquiries into the nature of interrogatives, indefinites, disjunction, and focus in Sinhala and other Languages: University of Illinois at Urbana-Champaign Ph. D. dissertation.

Szabolcsi, Anna. 2014a. Quantifier particles and compositionality. In Maria Aloni, Michael Franke \& Floris Roelofsen (eds.), Amsterdam Colloquium (AC) 19, 27-34.

Szabolcsi, Anna. 2014b. What do quantifier particles do? Ms., New York University. Uegaki, Wataru. 2014. Cross-linguistic variation in the derivation of alternative questions: Japanese and beyond. Ms., Massachusetts Institute of Technology. 
Wataru Uegaki

77 Massachusetts Avenue, Cambridge MA. 02139

Department of Linguistics and Philosophy, MIT wuegaki@mit.edu 